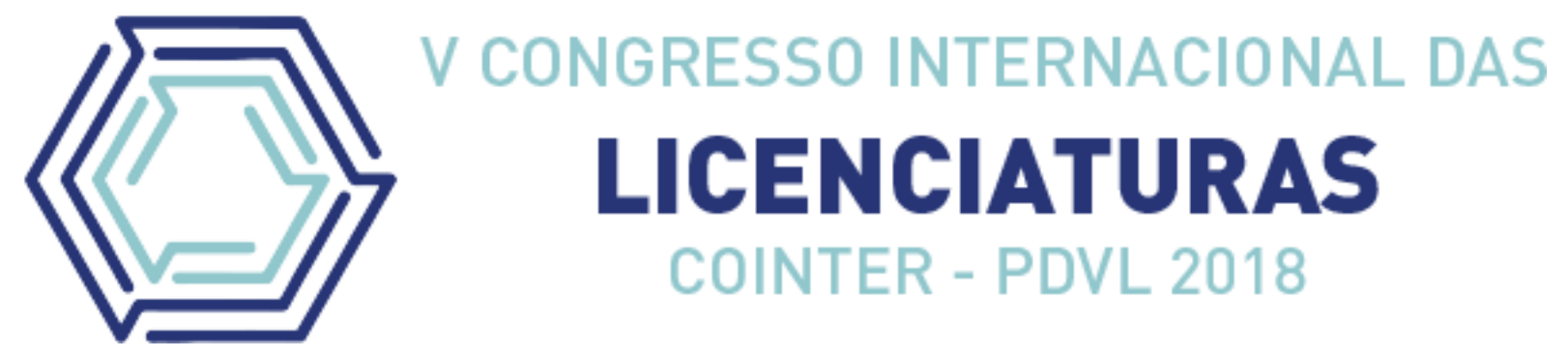

\title{
A CONSTRUÇÃO DE UM CALORÍMETRO COM MATERIAL DE BAIXO CUSTO: UM MODELO INSTRUMENTAL PARA MELHORIA DO ENSINO DE QUÍMICA
}

\section{THE CONSTRUCTION OF A CALORIMETER WITH LOW COST MATERIAL: AN INSTRUMENTAL MODEL TO IMPROVE CHEMISTRY EDUCATION}

\author{
Apresentação: Comunicação Oral \\ Mayzza Márcia Araújo do Nascimento ${ }^{1}$; Andrei Fellipe Veríssimo de Lima ${ }^{2}$. Francisco \\ Emanoel Ferreira de Almeida ${ }^{3}$.
}

DOI: $\underline{\text { https://doi.org/10.31692/2358-9728.VCOINTERPDVL.2018.00003 }}$

\section{Resumo}

Sabe-se que um recurso indispensável no ensino de Química é a experimentação, uma vez que a Química é uma ciência experimental. Porém, uma das problemáticas desse ensino é a ausência de laboratórios nas escolas. A maior parte das escolas no Brasil não dispõe de um espaço físico adequado para a realização de aulas práticas da disciplina de Química. Diante deste quadro, o professor deve adequar-se à realidade da escola, buscando estratégias que superem esta problemática. Uma alternativa é a construção e utilização de equipamentos alternativos, com materiais de fácil acesso e pouco custo, que possibilite o estudo dos conteúdos químicos nos laboratórios, ou até mesmo em sala de aula, fazendo deste último um laboratório alternativo. Diante do exposto, a relevância deste trabalho recai na necessidade dos docentes na área de ensino de Química em adaptar as aulas experimentais de modo a superar as fragilidades encontradas no cenário atual educacional. Assim, foi construído um calorímetro alternativo utilizando materiais de baixo custo e fácil obtenção, como um modelo instrumental para melhoria do ensino de Química. O calorímetro é um equipamento que nos permite estudar as transferências de energia na forma de calor. Através deste aparelho pode-se determinar, por exemplo, a entalpia de neutralização de reações ácido-base das substâncias. Com isso, este trabalho foi realizado em duas etapas: a montagem do equipamento e os testes de eficiência para determinação da capacidade térmica e da entalpia de neutralização para ácidos e bases. Baseando-se na teoria, o erro experimental para a entalpia de neutralização em relação ao valor teórico deste experimento, ficou em torno de $6 \%$ em ambos os testes, sendo um resultado bastante satisfatório para o estudo. Com base na montagem efetuada e nos testes realizados comprovou-se que o equipamento substitui o calorímetro comercial e pode ser utilizado nas aulas de Química, seja no laboratório, seja em sala de aula, possibilitando o estudo da teoria de maneira prática e de fácil execução.

Palavras-Chave: Ensino de Química, Materiais Alternativos, Calorímetro.

\footnotetext{
${ }^{1}$ Licenciatura em Química, IFPB campus João Pessoa, mayzzaaraujo.quim@hotmail.com

2 Licenciatura em Química, IFPB campus João Pessoa, prof.andreiverissimo@hotmail.com

${ }^{3}$ Prof. Doutor do curso de Licenciatura em Química, IFPB campus João Pessoa, emanoel.almeida@ifpb.edu.br
} 


\begin{abstract}
It is known that an indispensable resource in the teaching of Chemistry is experimentation, since Chemistry is an experimental science. However, one of the problems of this teaching is the absence of laboratories in schools. Most schools in Brazil do not have an adequate physical space for practical classes in the discipline of Chemistry. Faced with this picture, the teacher must adapt to the reality of the school, seeking strategies that overcome this problem. An alternative is the construction and use of alternative equipment, with materials that are easily accessible and inexpensive, that allows the study of chemical contents in laboratories, or even in the classroom, making the latter an alternative laboratory. In view of the above, the relevance of this work rests on the need of teachers in the area of Chemistry teaching to adapt the experimental classes in order to overcome the weaknesses found in the current educational scenario. Thus, an alternative calorimeter was constructed using materials of low cost and easy to obtain, as an instrumental model to improve the teaching of Chemistry. The calorimeter is equipment that allows us to study the transfer of energy in the form of heat. Through this apparatus one can determine, for example, the enthalpy of neutralization of acidbase reactions of the substances. This work was carried out in two stages: the assembly of the equipment and the efficiency tests to determine the thermal capacity and the enthalpy of neutralization for acids and bases. Based on the theory, the experimental error for the enthalpy of neutralization in relation to the theoretical value of this experiment was around $6 \%$ in both tests, being a very satisfactory result for the study. Based on the assembly carried out and the tests carried out, it was verified that the equipment replaces the commercial calorimeter and can be used in Chemistry classes, either in the laboratory or in the classroom, enabling the study of the theory in a practical and easy way.
\end{abstract}

Keywords: Teaching Chemistry, Alternative Materials, Calorimeter.

\title{
Introdução
}

Uma das críticas endêmicas no ensino de Química é a ausência de laboratórios nas escolas. A maior parte das escolas no Brasil não dispõe de um espaço físico adequado para a realização de aulas práticas da disciplina de Química. Não obstante, quando a escola possui um laboratório, por muitas vezes não possui os equipamentos mínimos necessários para o desenvolvimento de experimentos, devido à falta de recursos financeiros e/ou ao custo elevado destes materiais. Diante deste quadro, o professor deve adequar-se à realidade da escola, buscando estratégias que superem esta problemática.

Uma boa saída é a construção e utilização de equipamentos alternativos, com materiais de fácil acesso e pouco custo, que possibilite o estudo dos conteúdos químicos, que possam atender as necessidades dos laboratórios, bem como a possibilidade de transformar a sala de aula em um laboratório alternativo. Para isso, devem-se adaptar as aulas práticas, utilizando experimentos alternativos, que auxiliem nas aulas de Química. A experimentação quando somada a uma metodologia alternativa de ensino, além de contribuir significativamente na 
aprendizagem dos estudantes, sobrepõe a ausência e as limitações dos laboratórios nas escolas.

Para Hess (1997 apud CASTRO; ARAÚJO, 2012) realizar experimentos alternativos de Química utilizando reagentes, vidrarias, catalisadores e outros materiais de baixo custo e de fácil acesso é um desafio para muitos professores principalmente quando trabalham em instituições que possuem pouco ou nenhum recurso financeiro. Percebe-se que nem sempre o professor está preparado para esses problemas e por isso se faz necessária uma mudança pedagógica quanto à formação docente que promova avanços na organização e planejamento educacional, e proporcione transformações básicas no modo de pensar e atuar quanto docentes.

Diante do exposto, a relevância deste trabalho recai na necessidade dos profissionais docentes do ensino de Química em adaptar as aulas experimentais de modo a superar as fragilidades encontradas no cenário atual educacional.

Assim, foi construído e testado por este grupo de pesquisa um calorímetro alternativo, utilizando materiais de baixo custo e fácil obtenção, como um modelo instrumental para melhoria do ensino de Química.

\section{Fundamentação Teórica}

Sabe-se que um recurso indispensável no ensino de Química é a experimentação, uma vez que a Química é uma ciência experimental. Tal ferramenta torna-se relevante no processo de ensinoaprendizagem do discente, pois permite conhecer a Ciência de maneira palpável e menos abstrata, numa perspectiva que possibilite coadunar teoria e prática, saber empírico com saber científico, além de despertar no discente a curiosidade e um maior interesse para com a disciplina, desde que seja executada com fim científico e não somente ilustrativo.

No estudo da Química a execução de experimentos planejados constitui etapa essencial do processo de aprendizado. Segundo Rosito (2008), a utilização da experimentação é considerada para o ensino de Ciências como essencial para a aprendizagem científica. Destarte, é do professor a responsabilidade de reconhecer a importância do processo de planejamento e elaboração de registros relativos à atividade experimental proposta, e assim buscar a incorporação de tecnologias, estimulando a emissão de hipóteses como atividade central da investigação científica e mostrando a importância da discussão das hipóteses construídas durante a realização da atividade. 
Porém, um dos problemas relacionados à qualidade do ensino de Química é a ausência da experimentação. Santos e Maldaner (2010 apud CASTRO; ARAÚJO, 2012) afirmam que esta ausência está embasada em "crenças veiculadas no meio educacional", e tais crenças referem-se à falta de laboratórios nas escolas, deficiência destes espaços, traduzida pela falta de materiais e equipamentos necessários para a realização de atividades experimentais, equipamentos até a inexistência de orientação pedagógica adequada, além da grade curricular que oferta um número insuficiente de aulas, o que limita a realização de atividades experimentais no laboratório.

Sendo assim, se faz necessário modificar o que se entende por laboratório, e para isso o professor deve adaptar suas aulas, utilizando formas alternativas de ensino de modo a despertar o interesse, o raciocínio e o entendimento dos conceitos químicos. Uma maneira de viabilizar os experimentos nas escolas é a adaptação de equipamentos e materiais alternativos, que podem ser construídos com utensílios do cotidiano e de baixo custo (SILVA et al., 2017).

A experimentação de baixo custo representa uma alternativa cuja importância reside no fato de diminuir o custo operacional dos laboratórios e gerar menor quantidade de lixo químico (além de permitir que mais experiências sejam realizadas durante o ano letivo) (TORRICELLI, 2007).

Nesse sentido, cabe ao professor, principalmente o que se encontra no processo de formação, perceber essas problemáticas e superar as fragilidades eminentes desse sistema de ensino praticado, e as dificuldades impostas à realidade educacional.

A Química enquanto ciência destaca-se pela necessidade de aulas experimentais e dentro de seus vários campos de atuação existe um em especial que está relacionado com as trocas térmicas no processo reativo, que é a Termoquímica. Esta área de conhecimento é também muito explorada pelos professores durante suas aulas, pois aborda vários conceitos químicos e físicos que estão presentes no cotidiano dos discentes, porém nem sempre estes conceitos são identificados pelos estudantes. Esta dificuldade dos mesmos em visualizarem os fenômenos e associá-los ao seu cotidiano, resulta do distanciamento entre teoria e prática, havendo, portanto, a necessidade de implantação de instrumentos que auxiliem na construção destes conhecimentos. Pensando nesta problemática, foi desenvolvido o calorímetro alternativo para auxiliar no estudo da Termoquímica, como ferramenta de aprendizagem em aulas de Química. 
O calorímetro é um equipamento que nos permite estudar as transferências de energia na forma de calor e que segundo Atkins e Paula (2011) pode ser definido como "um recipiente onde ocorre um processo físico ou químico, um termômetro e um banho de água circundante”. Através deste aparelho pode-se determinar, por exemplo, a entalpia de neutralização de reações ácido-base das substâncias. Substâncias ácidas e básicas estão presentes no nosso dia a dia e o estudo dessas reações é bastante comum em Química.

Desta maneira, pretende-se utilizar este equipamento para auxiliar no estudo da Termoquímica para os diversos estudantes, das mais variadas formação em Química.

\section{Metodologia}

Este trabalho foi desenvolvido por um grupo de pesquisa pertencente ao PDV (Programa Despertando Vocações), fomentado pelo IFPB campus João Pessoa, composto por estudantes em formação inicial do curso de Licenciatura em Química da referida instituição, sob a orientação do professor da disciplina de Laboratório de Materiais Alternativos e FísicoQuímica. O objetivo desta pesquisa é a elaboração e otimização de equipamentos e práticas experimentais construídas com material de baixo custo, como forma de fomentar as aulas de Química.

A pesquisa apresenta uma abordagem qualitativa, a qual "se desenvolve numa situação natural; é rica em dados descritivos, tem um plano aberto e flexível e focaliza a realidade de forma complexa e contextualizada" (LAKATOS; MARCONI, 2004, p. 271). Bem como apresenta uma abordagem quantitativa que "caracteriza-se pelo emprego da quantificação tanto nas modalidades de coleta de informações quanto no tratamento delas por meio de técnicas estatísticas" (LAKATOS; MARCONI, 2004, p. 269).

Os calorímetros são sistemas isolados, classificados de acordo com as trocas térmicas. O calorímetro construído nesta pesquisa se aproximou de um sistema adiabático, ou seja, quando não há troca de calor entre o sistema e o ambiente. Ao utilizar o termo "aproximou" significa dizer que não há um sistema adiabático perfeito. $\mathrm{O}$ exemplo que melhor se aproxima é o vaso Dewar.

Para uma melhor compreensão sobre o funcionamento do Calorímetro foi realizado um estudo sobre Calorimetria, Leis da Termodinâmica, Condutividade térmica dos materiais e Entalpia de neutralização. Estes conteúdos químicos estão inseridos na disciplina de FísicoQuímica. Em seguida, realizou-se uma pesquisa prévia de quais materiais seriam necessários 
para a construção do equipamento. Especificamente para o Calorímetro foi necessário utilizar materiais isolantes (baixa condutividade térmica), uma vez que estamos trabalhando com um sistema isolado termicamente.

Uma vez obtidos os materiais houve a montagem do equipamento seguindo o esquema da Figura 1. Os materiais utilizados na construção do Calorímetro Alternativo foram: Madeira, Isopor, Poliuretano, Recipiente de vidro, Papel alumínio/emborrachado, Parafusos, Tubos PVC, Seringas, Termômetro, Motor Elétrico e Tinta branca (spray).

Figura 1: Esquema calorímetro. Fonte própria. 2018.

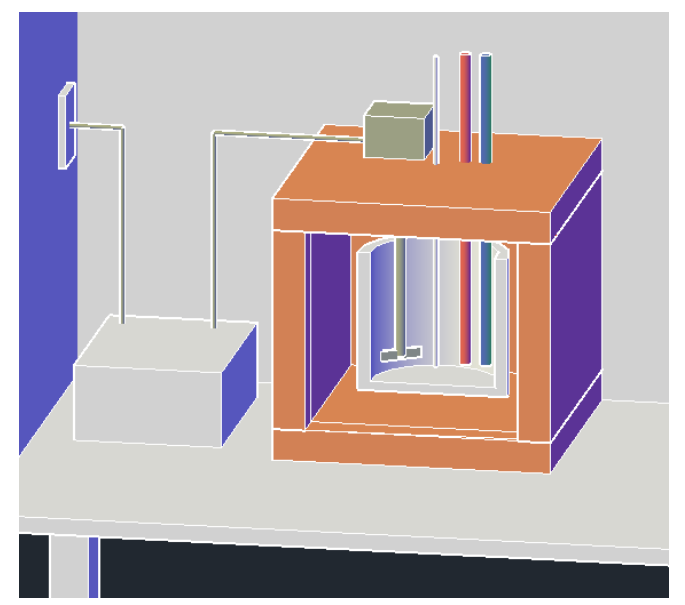

\section{Montagem do calorímetro}

A parte externa do calorímetro é uma caixa de madeira como base, que foi construída especificamente para este fim, e suas medidas são $173 \mathrm{~mm}$ x $127 \mathrm{~mm}$, e uma tampa de madeira. Para o sistema de isolamento (parte interna) utilizou-se uma caixa de isopor e espuma de poliuretano. O recipiente interno onde depositará os reagentes é um vidro de maionese reciclado, envolto por papel alumínio. Na tampa foram realizados furos para colocar o termômetro, os tubos de PVC onde serão introduzidos os reagentes com o auxílio das seringas, e o motor que foi adaptado para atuar como agitador mecânico.

De início, envolveu-se o vidro com papel alumínio e colocou-se na caixa de isopor. Em seguida, preencheu-se com a espuma de poliuretano. Pintou-se a caixa de madeira e a tampa, e depois se colocou o isopor. Já na tampa usou-se emborrachado na parte interna; na parte externa colocou-se o termômetro, o agitador e os tubos.

Após a montagem, foram realizados dois testes de eficiência no Calorímetro para verificar a funcionalidade do equipamento: determinação da capacidade térmica e determinação da entalpia de neutralização. Os roteiros utilizados nesta pesquisa foram adaptados do estudo realizado pelos autores Assumpção et al. (2010). 


\section{Testes de Eficiência}

\section{Determinação da capacidade térmica do calorímetro}

Procedimento

Mediu a temperatura do calorímetro $\left(\mathrm{T}_{\mathrm{c}}\right)$; Aferiu $150 \mathrm{~mL}$ de água destilada a temperatura ambiente. Anotou a temperatura $\left(\mathrm{T}_{\mathrm{f}}\right)$; Adicionou a água no calorímetro e fechou o sistema; Aqueceu $150 \mathrm{~mL}$ de água destilada até atingir $50^{\circ} \mathrm{C}\left(\mathrm{T}_{\mathrm{q}}\right)$; Adicionou rapidamente a água quente no calorímetro através das seringas; Verificou a temperatura a cada 30 segundos até atingir o equilíbrio $\left(\mathrm{T}_{\mathrm{e}}\right)$ e Calculou a capacidade térmica do calorímetro.

$O$ cálculo da capacidade térmica foi realizado através da equação $q=C .\left(T_{f}-T_{i}\right)$, para $\mathrm{C}=\mathrm{m}$. c, onde: $\mathrm{q}=$ calor transferido; $\mathrm{C}=$ capacidade térmica; $\mathrm{T}_{\mathrm{f}} \mathrm{e} \mathrm{T}_{\mathrm{i}}=$ temperaturas final $\mathrm{e}$ inicial; $m=$ massa; $c$ = calor específico da substância. ATKINS e PAULA (2011) relatam que uma vez que a quantidade de calor cedido pela água quente é igual à quantidade de calor recebido pelos componentes do sistema (considerando desprezível qualquer perda de calor para o ambiente), pode-se dizer que q (total) é igual à zero. Portanto: q (água fria) + q (água quente $)+\mathrm{q}$ (calorímetro) $=0$.

\section{Determinação da entalpia de neutralização}

Reagentes: Soluções de Ácido Clorídrico $\left(\mathrm{HCl}_{(\mathrm{aq})}\right)$, Ácido Acético $\left(\mathrm{H}_{3} \mathrm{CCOOH}_{(\mathrm{aq})}\right)$ e Hidróxido de Potássio (KOH), em concentrações 1,5 mol.L ${ }^{-1}$.

Para a determinação da entalpia, realizaram-se dois procedimentos: primeiro com o ácido clorídrico e o hidróxido de potássio (ácido forte e base forte); segundo com o ácido acético e o hidróxido de potássio (ácido fraco e base forte).

Procedimento

Mediu $100 \mathrm{~mL}$ de cada solução (ácido e base); Pesou em um béquer cada solução e anotou sua massa; Adicionou a solução básica no calorímetro, fechou o sistema e aguardou até atingir o equilíbrio $\left(\mathrm{T}_{\mathrm{c}}+\mathrm{T}_{\mathrm{b}}\right)$. Anotou a temperatura; Anotou a temperatura da solução ácida $\left(\mathrm{T}_{\mathrm{a}}\right)$; Adicionou a solução ácida no calorímetro através das seringas; Ligou o agitador e observou a temperatura máxima $\left(\mathrm{T}_{\mathrm{e}}\right)$.

$\mathrm{O}$ cálculo da entalpia de neutralização será realizado através da equação $\Delta \mathrm{H}=-[\mathrm{q}$ $($ calorímetro $)+\mathrm{q}($ sol. Ácida $)+\mathrm{q}($ sol. Básica $)] / \mathrm{n}$ (ácido ou base), onde: $\Delta \mathrm{H}=$ variação da entalpia; $\mathrm{q}=$ calor transferido; $\mathrm{n}$ = número de mols (ATKINS; PAULA, 2011). 


\section{Resultados e Discussão}

Para montagem deste equipamento realizou-se um estudo prévio de outros exemplos de calorímetros alternativos, sendo este otimizado para uma maior veracidade dos dados. Para o tratamento dos resultados utilizou-se do programa de gráficos, Orange, para uma melhor análise comportamental dos dados.

A Figura 2 mostra o resultado final do equipamento.

Figura 2: Calorímetro Alternativo. Fonte própria. 2018.

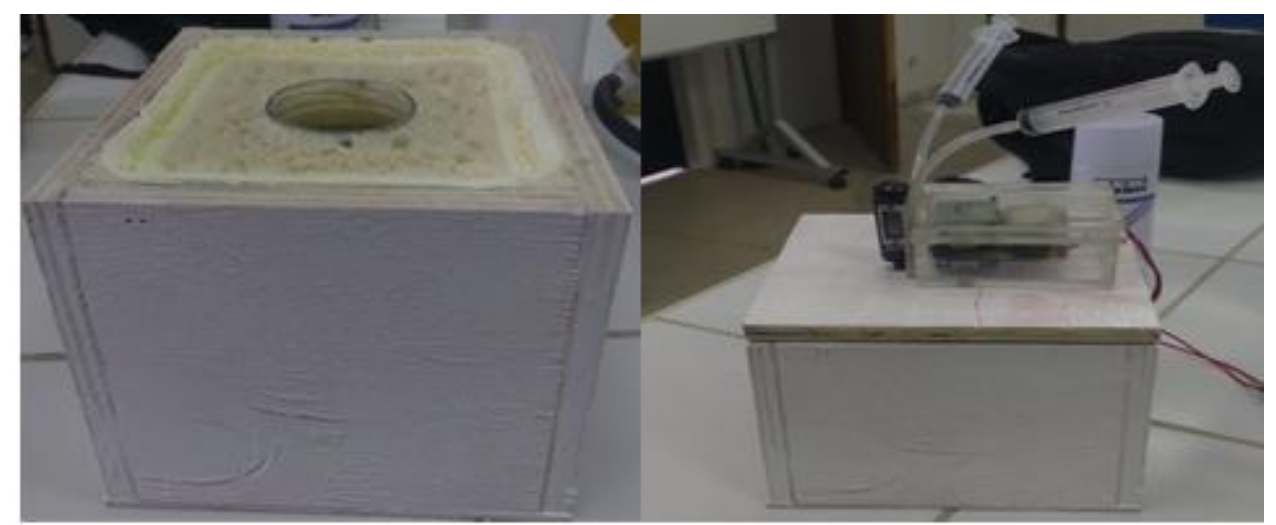

A seguir estão elencados os resultados referentes à determinação da Capacidade Térmica do Calorímetro e à determinação da Entalpia de Neutralização.

\section{Determinação da capacidade térmica do calorímetro}

A transferência de energia na forma de calor faz com que a temperatura de uma substância varie. Esta variação de temperatura da substância que absorve ou libera certa quantidade de energia é denominada capacidade térmica, $C=\frac{q}{\Delta T}$ (ATKINS; PAULA, 2011). Portanto, é preciso conhecer a capacidade térmica do calorímetro para determinar o calor liberado nas reações de neutralização. Esse procedimento consiste em aquecer o sistema e observar a variação de temperatura.

Para a determinação da capacidade térmica foi realizado o teste em triplicata. Os valores médios estão descritos abaixo.

Considerando-se que:

Densidade da água a $50{ }^{\circ} \mathrm{C}=0,986 \mathrm{~g} \mathrm{~mL}^{-1}$

Densidade da água a $25^{\circ} \mathrm{C}=0,997 \mathrm{~g} \mathrm{~mL}^{-1}$ Temos:

Massa da água fria $\left(150 \mathrm{~mL} .0,997 \mathrm{~g} \mathrm{~mL}^{-1}\right)=149,8 \mathrm{~g}$ 
Massa da água quente $\left(150 \mathrm{~mL}\right.$. 0,986 $\left.\mathrm{g} \mathrm{mL}^{-1}\right)=147,9 \mathrm{~g}$

Temperatura do calorímetro $\left(\mathrm{T}_{\mathrm{c}}\right)=24,0^{\circ} \mathrm{C}$

Temperatura da água fria $\left(\mathrm{T}_{\mathrm{f}}\right)=26,0^{\circ} \mathrm{C}$

Temperatura da água quente $\left(\mathrm{T}_{\mathrm{q}}\right)=50,0^{\circ} \mathrm{C}$

Temperatura equilíbrio (calorímetro + água fria + água quente) $\left(\mathrm{T}_{\mathrm{e}}\right)=31,1{ }^{\circ} \mathrm{C}$

Capacidade calorífica da água (c) $=4,18 \mathrm{~J} / \mathrm{g}{ }^{\circ} \mathrm{C}$

Calculando a capacidade térmica:

$$
\begin{gathered}
\mathrm{q} \text { (calorímetro) }+\mathrm{q}(\text { água fria })+\mathrm{q} \text { (água quente })=0 \\
\mathrm{C} \cdot\left(\mathrm{T}_{\mathrm{e}}-\mathrm{T}_{\mathrm{c}}\right)+\mathrm{m} \text { (água quente) } . \mathrm{c} \cdot\left(\mathrm{T}_{\mathrm{e}}-\mathrm{T}_{\mathrm{q}}\right)+\mathrm{m} \text { (água fria) } . \mathrm{c} \cdot\left(\mathrm{T}_{\mathrm{e}}-\mathrm{T}_{\mathrm{f}}\right)=0 \\
\mathrm{C} \cdot(31,1-24,0)+147,9 \cdot 4,18 \cdot(31,1-50)+149,8 \cdot 4,18 \cdot(31,1-26,0)=0
\end{gathered}
$$

Capacidade térmica do calorímetro $=119,59 \mathrm{~J} /{ }^{\circ} \mathrm{C}$

\section{Determinação da entalpia de neutralização}

Em 1887, Arrhenius propôs que em soluções eletrolíticas existe um equilíbrio entre as moléculas não dissociadas de um soluto e os íons que delas provêm pela dissociação eletrolítica. "Os ácidos e as bases fortes sendo quase que totalmente dissociados, sua interação era, em todos os casos, equivalente a $H^{+}(a q)+\mathrm{OH}^{-}(a q) \rightleftharpoons \mathrm{H}_{2} O(l)$, explicando assim a constância do valor da entalpia de neutralização dos mesmos” (ASSUMPÇÃO et al, p. 64, 2010).

A entalpia de neutralização, reação ácido-base, é definida como o calor liberado durante a neutralização de uma solução diluída de ácido por uma solução diluída de base. Segundo Arrhenius, essa entalpia de neutralização é sempre igual a - 55,84 kJ.mol ${ }^{-1}$, a $2{ }^{\circ} \mathrm{C}$ (ASSUMPÇÃO et al, 2010).

Ao calcularmos o $\Delta \mathrm{H}$ da reação de neutralização entre o $\mathrm{HCl}_{(\mathrm{aq})}+\mathrm{NaOH}_{(\mathrm{aq})}$, a partir das entalpias de formação, temos:

$$
\begin{aligned}
& \mathrm{H}_{(a q)}^{+}+\mathrm{Cl}_{(a q)}^{-}+\mathrm{Na}_{(a q)}+\mathrm{OH}_{(a q)}^{-} \rightleftharpoons \mathrm{Na}_{(a q)}^{+}+\mathrm{Cl}_{(a q)}^{-}+\mathrm{H}_{2} \mathrm{O}_{(\mathrm{l})} \\
& \Delta H r=\sum H_{f}(p)-\sum H_{f}(r) \\
& \Delta H r=\left\{H_{f}\left(\mathrm{Na}_{(a q)}^{+}\right)+H_{f}\left(\mathrm{Cl}_{(a q)}^{-}\right)+H_{f}\left(H_{2} \mathrm{O}_{(\mathrm{l})}\right)\right\}-\left\{H_{f}\left(\mathrm{H}^{+}{ }_{(a q)}\right)+\mathrm{H}_{f}\left(\mathrm{Cl}^{-}{ }_{(a q)}\right)\right. \\
& \left.+\mathrm{H}_{f}\left(\mathrm{Na}_{(a q)}^{+}\right)+\mathrm{H}_{f}\left(\mathrm{OH}_{(\mathrm{aq})}^{-}\right)\right\} \\
& \Delta H r=\{(-240,12)+(-167,16)+(-285,83)\}-\{(0)+(-167,16)+(-240,12)+(-229,99)\} \\
& \Delta \mathrm{Hr}=-55,84 \mathrm{~kJ} \cdot \mathrm{mol}^{-1}
\end{aligned}
$$

* $\mathrm{H}_{\mathrm{f}}$ é a entalpia de formação; $\Delta \mathrm{H}$ é a variação da entalpia de neutralização da reação. 
A reação de neutralização é um processo exotérmico (libera calor), e quando ocorre em um sistema adiabático, a temperatura do sistema se eleva. Em resumo, a variação de entalpia $(\Delta \mathrm{H})$ é negativa.

Para a determinação da entalpia de neutralização ácido-base temos os seguintes dados:

a) Ácido forte e Base forte

$$
\mathrm{HCl}_{(a q)}+\mathrm{KOH}_{(a q)} \rightleftharpoons \mathrm{KCl}_{(a q)}+\mathrm{H}_{2} \mathrm{O}_{(\mathrm{l})}
$$

Massa do hidróxido de potássio $=106,86 \mathrm{~g}$

Massa do ácido clorídrico $=102,38 \mathrm{~g}$

Temperatura da base $\left(T_{b}\right)+$ Temperatura do calorímetro $\left(T_{c}\right)=26,5^{\circ} \mathrm{C}$

Temperatura do ácido $\left(\mathrm{T}_{\mathrm{a}}\right)=26,4^{\circ} \mathrm{C}$

Temperatura de equilíbrio (temperatura máxima observada) $\left(\mathrm{T}_{\mathrm{e}}\right)=35,4{ }^{\circ} \mathrm{C}$

$\mathrm{n}_{\mathrm{OH}}($ base $)=0,15 \mathrm{~mol}$

Capacidade calorífica da água $(\mathrm{c})=4,18 \mathrm{~J} / \mathrm{g}{ }^{\circ} \mathrm{C}$ (considera-se a capacidade calorífica da água, pois os reagentes, ácido e base, estão em solução).

Calculando a entalpia de neutralização:

$\Delta \mathrm{H}=-[\mathrm{q}$ (calorímetro $)+\mathrm{q}($ sol. Ácida $)+\mathrm{q}$ (sol. Básica) $]$ / n (ácido ou base $)$

$$
\Delta \mathrm{H}=-\left[\mathrm{C} .\left(\mathrm{T}_{\mathrm{e}}-\mathrm{T}_{\mathrm{c}}\right)+\mathrm{m} \text { (sol. Ácida) . c . }\left(\mathrm{T}_{\mathrm{e}}-\mathrm{T}_{\mathrm{a}}\right)+\mathrm{m} \text { (sol. Básica) . c . }\left(\mathrm{T}_{\mathrm{e}}-\mathrm{T}_{\mathrm{b}}\right)\right] / \mathrm{n}_{\mathrm{OH}}
$$$$
\Delta \mathrm{H}=-[\mathbf{1 1 9 , 5 9} .(35,4-26,5)+102,38 \text {. 4,18 . }(35,4-26,4)+106,86.4,18 .(35,4-26,5)] /
$$

$$
\Delta \mathbf{H}=\mathbf{5 9 , 3} \mathrm{kJ} / \mathrm{mol}
$$

b) Ácido fraco e Base forte

$$
\mathrm{CH}_{3} \mathrm{COOH}_{(a q)}+\mathrm{KOH}_{(a q)} \rightleftharpoons \mathrm{KCH}_{3} \mathrm{COO}_{(a q)}+\mathrm{H}_{2} \mathrm{O}_{(l)}
$$

Massa do hidróxido de potássio $=107,38 \mathrm{~g}$

Massa do ácido acético $=95,37 \mathrm{~g}$

Temperatura da base $\left(\mathrm{T}_{\mathrm{b}}\right)+$ Temperatura do calorímetro $\left(\mathrm{T}_{\mathrm{c}}\right)=26,2^{\circ} \mathrm{C}$

Temperatura do ácido $\left(\mathrm{T}_{\mathrm{a}}\right)=26,4^{\circ} \mathrm{C}$

Temperatura de equilíbrio (temperatura máxima observada) $\left(\mathrm{T}_{\mathrm{e}}\right)=34,4^{\circ} \mathrm{C}$

$\mathrm{n}_{\mathrm{OH}}($ base $)=0,15 \mathrm{~mol}$

Capacidade calorífica da água $(\mathrm{c})=4,18 \mathrm{~J} / \mathrm{g}{ }^{\circ} \mathrm{C}$ (considera-se a capacidade calorífica da água, pois os reagentes, ácido e base, estão em solução). 
Calculando a entalpia de neutralização:

$$
\begin{gathered}
\Delta \mathrm{H}=-[\mathrm{q} \text { (calorímetro })+\mathrm{q}(\text { sol. Ácida })+\mathrm{q}(\text { sol. Básica })] / \mathrm{n} \text { (ácido ou base }) \\
\Delta \mathrm{H}=-\left[\mathrm{C} .\left(\mathrm{T}_{\mathrm{e}}-\mathrm{T}_{\mathrm{c}}\right)+\mathrm{m}(\text { sol. Ácida }) . \mathrm{c} .\left(\mathrm{T}_{\mathrm{e}}-\mathrm{T}_{\mathrm{a}}\right)+\mathrm{m}(\text { sol. Básica }) . \mathrm{c} .\left(\mathrm{T}_{\mathrm{e}}-\mathrm{T}_{\mathrm{b}}\right)\right] / \mathrm{n}_{\mathrm{OH}} \\
\Delta \mathrm{H}=-[\mathbf{1 1 9 , 5 9} .(34,4-26,2)+95,37.4,18 .(34,4-26,4)+107,38.4,18 .(34,4-26,2)] / \\
0,15 \\
\Delta \mathbf{H}=\mathbf{- 5 2 , 4} \mathbf{~ k J} / \mathbf{m o l}
\end{gathered}
$$

Com base na montagem efetuada e nos testes realizados, comprovou-se que o equipamento substitui o calorímetro comercial e permite o estudo da Termoquímica nas aulas, uma vez que, baseando-se na teoria, o erro experimental da entalpia de neutralização em relação ao valor teórico ficou em torno de $6 \%$ em ambos os testes, possibilitando o estudo da teoria de maneira prática e de fácil execução.

\section{Conclusões}

Os problemas atualmente vivenciados no ensino de Química revelam a necessidade de práticas inovadoras, sendo importante a busca por novas ferramentas didáticas para despertar o interesse dos discentes. O experimento supracitado pode ser contextualizado com situações do dia a dia, além de explorar vários conteúdos químicos de diferentes seguimentos da Química. Vale ressaltar que é possível implementar um experimento simples e de baixo custo, mas de grande importância pedagógica.

Podemos concluir com este estudo que é possível construir um calorímetro alternativo elaborado com material de baixo custo que permita a sua aplicabilidade em aulas experimentais, visando à melhoria do ensino através de uma melhor absorção do conhecimento, pois os resultados adquiridos com o dispositivo elaborado apresentou um erro experimental em torno de $6 \%$ para a entalpia de neutralização nos testes realizados. $\mathrm{O}$ erro entre o valor calculado e o teórico pode estar associado aos seguintes fatores: ao próprio sistema que não é totalmente isolado e erros associados, normalmente, à coleta de dados.

Podemos afirmar que o equipamento possibilita o estudo da entalpia de neutralização e pode ser utilizado facilmente nas aulas de Química. Outro ponto importante é o baixo custo da elaboração do equipamento se comparado com os equipamentos comerciais.

\section{Referências}


ASSUMPÇÃO, M. H. M. T. et al. Construção de um calorímetro de baixo custo para a determinação de entalpia de neutralização. Eclética Química, v. 35, n. 2, p. 63-69, 2010.

ATKINS, P.; PAULA, J. Físico-Química: fundamentos. 5ª ed. Rio de Janeiro. 2011.

CASTRO, C. L.; ARAÚJO, S. C. M. Uma proposta De Experimentos Com Materiais Alternativos a partir da Análise Do Livro Didático. In XVI Encontro Nacional de Ensino de Química (XVI ENEQ) e X Encontro de Educação Química da Bahia (X EDUQUI) Salvador - BA. Julho/2012.

LAKATOS, E. M.; MARCONI, M. A. Metodologia Científica. $4^{\mathrm{a}}$ ed. São Paulo: Atlas, 2004.

ROSITO, B. A. O Ensino de Ciências e a Experimentação. In: MORAES, R. (org.). Construtivismo e Ensino de Ciências: Reflexões Epistemológicas e Metodológicas. Porto Alegre: EDIPUCRS, 2008.

SILVA, J. N.; et al. Experimentos de baixo custo aplicados ao ensino de química: contribuição ao processo ensino-aprendizagem. Scientia Plena. Vol. 13, N. 01. 2017.

TORRICELLI, E. Dificuldades de aprendizagem no Ensino de Química. (Tese de livre docência), Belo Horizonte, Universidade Federal de Minas Gerais. Faculdade de Educação, 2007. 